\title{
Degenerate Periodic Orbits and Homoclinic Torus Bifurcation
}

\author{
Thomas J. Bridges* and Neil M. Donaldson ${ }^{\dagger}$ \\ Department of Mathematics, University of Surrey, Guildford, Surrey GU2 7XH England, United Kingdom
}

(Received 20 June 2005; published 1 September 2005)

\begin{abstract}
A one-parameter family of periodic orbits with frequency $\omega$ and energy $E$ of an autonomous Hamiltonian system is degenerate when $E^{\prime}(\omega)=0$. In this paper, new features of the nonlinear bifurcation near this degeneracy are identified. A new normal form is found where the coefficient of the nonlinear term is determined by the curvature of the energy-frequency map. An important property of the bifurcating "homoclinic torus" is the homoclinic angle and a new asymptotic formula for it is derived. The theory is constructive, and so is useful for physical applications and in numerics.
\end{abstract}

DOI: 10.1103/PhysRevLett.95.104301

PACS numbers: 45.20.Jj, 05.45.-a

Introduction. - Periodic solutions of Hamiltonian systems are important in a wide range of physical phenomena: examples are celestial mechanics [1-4], oscillator networks [5], quantum chaos [6-8], and water waves [9,10]. In autonomous Hamiltonian systems, periodic orbits arise in one-parameter families, parameterized by the energy [11]. In this Letter, a new theory is presented for the nonlinear behavior when a periodic orbit is degenerate.

Consider a nonlinear autonomous Hamiltonian system

$$
\mathbf{q}_{t}=\frac{\partial H}{\partial \mathbf{p}}, \quad \mathbf{p}_{t}=-\frac{\partial H}{\partial \mathbf{q}}, \quad(\mathbf{q}, \mathbf{p}) \in \mathbb{R}^{4},
$$

where $H(\mathbf{q}, \mathbf{p})$ is a given smooth Hamiltonian function. (Attention is restricted to 4-dimensional phase space as it is the lowest dimension in which the bifurcation occurs.) Suppose there exists a one-parameter family of periodic solutions,

$$
(\mathbf{q}(t), \mathbf{p}(t)):=(\hat{\mathbf{q}}(\theta, \omega), \hat{\mathbf{p}}(\theta, \omega)), \quad \theta=\omega t+\theta_{0},
$$

where $\omega>0$ is the frequency of the periodic orbit, $\theta_{0}$ is an arbitrary phase shift, and $(\hat{\mathbf{q}}, \hat{\mathbf{p}})$ are $2 \pi$-periodic functions of $\theta$. Substituting (2) in (1) shows that the periodic orbit satisfies

$$
-\omega \hat{\mathbf{p}}_{\theta}=\frac{\partial H}{\partial \mathbf{q}}(\hat{\mathbf{q}}, \hat{\mathbf{p}}), \quad \omega \hat{\mathbf{q}}_{\theta}=\frac{\partial H}{\partial \mathbf{p}}(\hat{\mathbf{q}}, \hat{\mathbf{p}}) .
$$

Here and henceforth subscripts (other than integers) will indicate differentiation with respect to that parameter.

Let $E(\omega)=H(\hat{\mathbf{q}}(\theta, \omega), \hat{\mathbf{p}}(\theta, \omega))$. A periodic orbit on the branch of periodic solutions is nondegenerate when $E^{\prime}(\omega) \neq 0$. Because of the importance of the $E-\omega$ relationship, it is customary to plot branches of periodic solutions in the $(E, \omega)$ plane as shown in Fig. 1.

At points where $E^{\prime}(\omega)$ changes sign, a pair of Floquet multipliers coalesces at +1 . This bifurcation was first observed by Poincaré and is widely used in practice (see [1] for a history and examples). A proof that $E^{\prime}(\omega)=0$ signals a bifurcation of Floquet multipliers is given in Theorem 5(iii) of [5]. A schematic of this bifurcation is shown in Fig. 1.
At points where the energy is stationary, the action is also stationary, $\mathcal{A}^{\prime}(\omega)=0$, where the action of a family of periodic orbits is defined by

$$
\mathcal{A}(\omega)=\oint \hat{\mathbf{p}} \cdot d \hat{\mathbf{q}}:=\frac{1}{2 \pi} \int_{0}^{2 \pi} \hat{\mathbf{p}} \cdot \hat{\mathbf{q}}_{\theta} d \theta .
$$

Differentiating the energy of a periodic orbit and using (3) shows that $E^{\prime}(\omega)=\omega \mathcal{A}^{\prime}(\omega)$. Moreover, $\quad E^{\prime \prime}(\omega)=$ $\omega \mathcal{A}^{\prime \prime}(\omega)$ at points where $E^{\prime}(\omega)=0$.

The aim of this Letter is to describe the nonlinear behavior in the phase space when the periodic orbit passes through a degeneracy. Our main result is that there exists new coordinates $(\phi, u, I, v)$ satisfying

$$
\begin{aligned}
& -I_{t}=0 \quad-v_{t}=I+\delta u-\frac{1}{2} \sigma u^{2}+\cdots \\
& \phi_{t}=u+\cdots \quad u_{t}=\varepsilon v+\cdots .
\end{aligned}
$$

The most remarkable feature of this normal form is the role of the curvature of the energy-frequency curve

$$
\sigma=a^{3} \mathcal{A}^{\prime \prime}\left(\omega_{0}\right)=\frac{a^{3}}{\omega_{0}} E^{\prime \prime}\left(\omega_{0}\right),
$$

where $a$ is a strictly positive (explicitly computable) constant. The other parameters $\delta, \varepsilon$ and the coordinates will all be explicitly determined in the sequel. The theory is con-

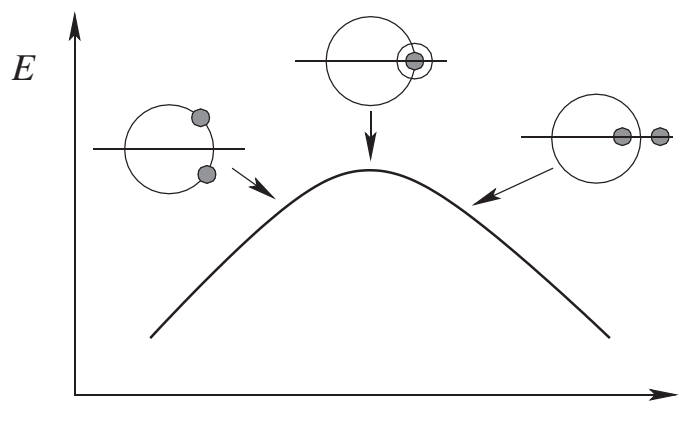

$\omega$

FIG. 1. Schematic of the induced bifurcation of Floquet multipliers when $E^{\prime}(\omega)$ changes sign. 
structive and uses only geometric properties of the family of periodic orbits. The normal form (5) is completely integrable and so a complete analysis of its solutions can be obtained.

There is a sequence of observations and steps which lead to this result. First, when a pair of Floquet multipliers coalesces at +1 in a Hamiltonian system linearized about a periodic orbit, there is an attendant homoclinic bifurcation (cf. Section 7.4 and Fig. 80 in Ref. [2]); see also [12] for the related problem near an equilibrium.

However, this existing theory is incomplete and for some applications it is lacking crucial information. Firstly, the reduction to a plane or use of a Poincaré section eliminates phase information which is important in a number of contexts. For example, this phase, called the "homoclinic angle" in [6], has been shown to be important in semiclassical quantization. The phase is also important for wave problems $[9,10]$ and is essential when computing branches of orbits which are homoclinic to periodic manifolds $[13,14]$. Secondly, the features of the new normal form are determined by geometric properties of the periodic orbit. Thirdly, the theory is constructive.

The phase shift is a crucial part of the dynamics near the degenerate periodic orbit. The bifurcating homoclinic orbit in (5) leaves the unstable manifold of the periodic orbit and reenters along the stable manifold, shifted by a phase, as shown schematically in Fig. 2. The asymptotic behavior of the phase shift is found here to satisfy

$$
\Delta \phi \sim\left|E(\omega)-E\left(\omega_{0}\right)\right|^{1 / 4}, \quad \text { as } \omega \rightarrow \omega_{0} .
$$

The applications of (5) are multifold. The authors' motivation for this theory is water waves: this bifurcation arises in the analysis of wave breaking [9] and is the starting point

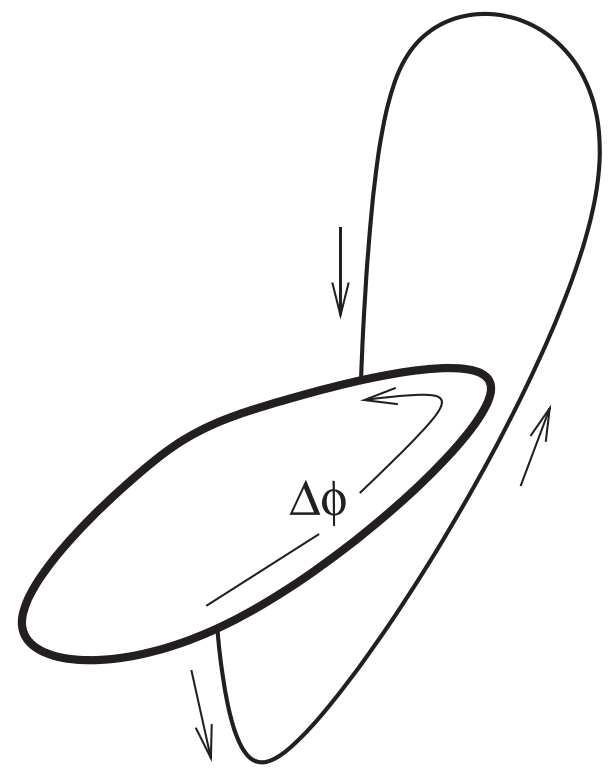

FIG. 2. Schematic of the phase shift of the orbit which is homoclinic to the periodic manifold. for a branch of steady dark solitary waves in shallow water [10]. A simple example is included in the last section to demonstrate the constructive aspects of the theory.

Linearization about a degenerate periodic orbit. - Let $\hat{U}(\theta, \omega)=(\hat{\mathbf{q}}(\theta, \omega), \hat{\mathbf{p}}(\theta, \omega))$, to streamline the derivation, and represent (3) as

$$
\nabla H(\hat{U})=\omega \mathbf{J} \hat{U}_{\theta}, \quad \mathbf{J}=\left[\begin{array}{cc}
\mathbf{0} & -\mathbf{I} \\
\mathbf{I} & \mathbf{0}
\end{array}\right] .
$$

The linearization about a periodic orbit always has a Floquet multiplier at +1 of geometric multiplicity one and algebraic multiplicity two. This property follows from differentiation of (8) with respect to $\theta$ and $\omega$,

$$
\mathbf{L}(\theta) \hat{\xi}_{1}=0 \quad \text { and } \quad \mathbf{L}(\theta) \hat{\xi}_{2}=\mathbf{J} \hat{\xi}_{1} \text {, }
$$

with $\hat{\xi}_{1}=\hat{U}_{\theta}, \hat{\xi}_{2}=\hat{U}_{\omega}$ and

$$
\mathbf{L}(\theta):=D^{2} H(\hat{U}(\theta))-\omega \mathbf{J} \frac{\partial}{\partial \theta} .
$$

The consequence of the degeneracy $E^{\prime}(\omega)=0$ is that the algebraic multiplicity of the Floquet multiplier +1 is increased to at least four [5]. When the periodic orbit is degenerate, there exist functions

$$
\mathbf{L}(\theta) \hat{\xi}_{3}(\theta)=\mathbf{J} \hat{\xi}_{2}(\theta) \quad \text { and } \quad \mathbf{L}(\theta) \hat{\xi}_{4}(\theta)=\mathbf{J} \hat{\xi}_{3}(\theta) .
$$

If $\mathbf{L}(\theta) \hat{\xi}_{5}(\theta)=\mathbf{J} \hat{\xi}_{4}(\theta)$ is not solvable, the algebraic multiplicity is exactly four, that is, $\left\langle\left\langle\hat{\xi}_{1}, \mathbf{J} \hat{\xi}_{4}\right\rangle\right\rangle \neq 0$ where $\langle\langle\cdot, \cdot\rangle\rangle$ is defined in

$$
\left\langle\left\langle\hat{\xi}_{1}, \mathbf{J} \hat{\xi}_{4}\right\rangle\right\rangle:=\frac{1}{2 \pi} \int_{0}^{2 \pi}\left\langle\hat{\xi}_{1}, \mathbf{J} \hat{\xi}_{4}\right\rangle d \theta,
$$

The solutions $\hat{\xi}_{j}(\theta), j=1,2,3,4$ are not unique. More importantly, they do not form a symplectic basis. Therefore introduce the normalized basis

$$
\begin{aligned}
& \xi_{1}(\theta)=a \hat{\xi}_{1}(\theta), \quad \xi_{2}(\theta)=a \hat{\xi}_{2}(\theta), \\
& \xi_{3}(\theta)=a \hat{\xi}_{3}(\theta)+b \hat{\xi}_{1}(\theta), \quad \xi_{4}(\theta)=a \hat{\xi}_{4}(\theta)+b \hat{\xi}_{2}(\theta) . \\
& \text { where } \varepsilon=\operatorname{sign}\left\langle\left\langle\mathbf{J} \hat{\xi}_{4}, \hat{\xi}_{1}\right\rangle\right\rangle, \\
& \qquad a=\left|\left\langle\left\langle\mathbf{J} \hat{\xi}_{4}, \hat{\xi}_{1}\right\rangle\right\rangle\right|^{-1 / 2} \quad \text { and } \quad b=-\frac{1}{2} \varepsilon a^{3}\left\langle\left\langle\hat{\xi}_{3}, \mathbf{J} \hat{\xi}_{4}\right\rangle\right\rangle .
\end{aligned}
$$

This set of vectors still forms a Jordan chain:

$$
\begin{aligned}
& \mathbf{L} \boldsymbol{\xi}_{1}=0, \quad \mathbf{L} \boldsymbol{\xi}_{2}=\mathbf{J} \boldsymbol{\xi}_{1}, \quad \mathbf{L} \boldsymbol{\xi}_{3}=\mathbf{J} \boldsymbol{\xi}_{2}, \\
& \mathbf{L} \boldsymbol{\xi}_{4}=\mathbf{J} \xi_{3},
\end{aligned}
$$

but they will provide a symplectic basis since

$$
\left\{\left\langle\left\langle\boldsymbol{\xi}_{i}, \mathbf{J} \boldsymbol{\xi}_{j}\right\rangle\right\rangle\right\}=\left(\begin{array}{cccc}
0 & 0 & 0 & \varepsilon \\
0 & 0 & -\varepsilon & 0 \\
0 & \varepsilon & 0 & 0 \\
-\varepsilon & 0 & 0 & 0
\end{array}\right) .
$$


The appropriate ordering of the basis is therefore $\left\{\boldsymbol{\xi}_{1}, \boldsymbol{\xi}_{2},-\varepsilon \boldsymbol{\xi}_{4}, \varepsilon \boldsymbol{\xi}_{3}\right\}$. This basis suggests a coordinate transformation of the form $U(t)=\hat{U}+V$, with

$$
\begin{aligned}
V= & \phi(t) \xi_{1}(\theta)+u(t) \xi_{2}(\theta)-\varepsilon I(t) \xi_{4}(\theta)+\varepsilon v(t) \xi_{3}(\theta) \\
& +\cdots
\end{aligned}
$$

Substituting $V$ into the linearization of (1) leads to

$$
-\dot{I}=0, \quad-\dot{v}=I, \quad \dot{\phi}=u, \quad \dot{u}=\varepsilon v,
$$

a linear Hamiltonian system with $H=u I+\frac{1}{2} \varepsilon v^{2}$ and symplectic form $d I \wedge d \phi+d v \wedge d u$.

Further properties of the linearization. - There are additional properties of the linearization which will be essential for determining the critical coefficient of the nonlinear term in the normal form. Differentiating (9),

$$
\begin{aligned}
& \mathbf{L}(\theta) \hat{U}_{\theta \theta}=-D^{3} H\left(\hat{U}_{\theta}, \hat{U}_{\theta}\right) \\
& \mathbf{L}(\theta) \hat{U}_{\theta \omega}=-D^{3} H\left(\hat{U}_{\theta}, \hat{U}_{\omega}\right)+\mathbf{J} \hat{U}_{\theta \theta} \\
& \mathbf{L}(\theta) \hat{U}_{\omega \omega}=-D^{3} H\left(\hat{U}_{\omega}, \hat{U}_{\omega}\right)+2 \mathbf{J} \hat{U}_{\theta \omega} .
\end{aligned}
$$

Expressing the action in (4) as $\frac{1}{2}\left\langle\left\langle\mathbf{J} \hat{U}_{\theta}, \hat{U}\right\rangle\right\rangle$, it follows that $A^{\prime}(\omega)=\left\langle\left\langle\mathbf{J} \hat{U}_{\theta}, \hat{U}_{\omega}\right\rangle\right\rangle$, and

$$
\mathcal{A}{ }^{\prime \prime}(\omega)=\left\langle\left\langle\mathbf{J} \hat{U}_{\theta}, \hat{U}_{\omega \omega}\right\rangle\right\rangle+\left\langle\left\langle\mathbf{J} \hat{U}_{\theta \omega}, \hat{U}_{\omega}\right\rangle\right\rangle .
$$

Now, using the above formulas (14),

$$
\begin{aligned}
\left\langle\left\langle\mathbf{J} \hat{U}_{\theta \omega}, \hat{U}_{\omega}\right\rangle\right\rangle= & -\frac{1}{a}\left\langle\left\langle\hat{U}_{\theta \omega}, \mathbf{J} \boldsymbol{\xi}_{2}\right\rangle\right\rangle=-\frac{1}{a}\left\langle\left\langle\hat{U}_{\theta \omega}, \mathbf{L}(\theta) \boldsymbol{\xi}_{3}\right\rangle\right\rangle \\
= & -\frac{1}{a}\left\langle\left\langle\mathbf{L}(\theta) \hat{U}_{\theta \omega}, \boldsymbol{\xi}_{3}\right\rangle\right\rangle \\
= & \frac{1}{a^{3}}\left\langle\left\langle D^{3} H\left(\boldsymbol{\xi}_{1}, \boldsymbol{\xi}_{2}\right), \boldsymbol{\xi}_{3}\right\rangle\right\rangle+\frac{1}{a}\left\langle\left\langle\hat{U}_{\theta \theta}, \mathbf{J} \boldsymbol{\xi}_{3}\right\rangle\right\rangle \\
= & \frac{1}{a^{3}}\left\langle\left\langle D^{3} H\left(\boldsymbol{\xi}_{1}, \boldsymbol{\xi}_{2}\right), \boldsymbol{\xi}_{3}\right\rangle\right\rangle+\frac{1}{a} \\
& \times\left\langle\left\langle\hat{U}_{\theta \theta}, \mathbf{L}(\theta) \boldsymbol{\xi}_{4}\right\rangle\right\rangle \\
= & \frac{1}{a^{3}}\left\langle\left\langle D^{3} H\left(\boldsymbol{\xi}_{1}, \boldsymbol{\xi}_{2}\right), \boldsymbol{\xi}_{3}\right\rangle\right\rangle-\frac{1}{a^{3}} \\
& \times\left\langle\left\langle D^{3} H\left(\boldsymbol{\xi}_{1}, \boldsymbol{\xi}_{1}\right), \boldsymbol{\xi}_{4}\right\rangle\right\rangle,
\end{aligned}
$$

which will be useful when computing $\sigma$ in (5).

Geometric properties of nonlinear normal form. - Given the linear Hamiltonian system (13), the nonlinear normal form to all orders has been given in [15]: see Example 2 in that paper, with $\left(\xi_{1}, \xi_{2}, \eta_{1}, \eta_{2}\right) \mapsto(I, v, \phi, u)$, and the affine symmetry $\phi \mapsto \phi+c$. The normalized Hamiltonian function is of the form

$$
\begin{aligned}
& H=I u+\frac{1}{2} \varepsilon v^{2}+F\left(u^{2}, \tau\right)+u^{3} G\left(u^{2}, \tau\right), \\
& \tau=3 u^{2} v^{2}+8 \varepsilon I u^{3}
\end{aligned}
$$

for some smooth functions $F$ and $G$. Expanding $F$ and $G$ in Taylor series, the nonlinear normal form to leading order is as given in (5), with ... representing nonlinear terms of degree 3 and higher. The unfolding parameter $\delta$ is not qualitatively significant and so is ignored in the local analysis. It remains to find a formula for $\sigma$.

Write the perturbed solution near the degenerate periodic orbit as $U(t)=\hat{U}(\theta, \omega)+V(\theta, t)$. Then $V$ satisfies

$$
\begin{aligned}
\mathbf{J} V_{t} & =\nabla H(\hat{U}+V)-\nabla H(\hat{U})-\omega \mathbf{J} V_{\theta} \\
& =\mathbf{L}(\theta) V+\frac{1}{2} D^{3} H(\hat{U})(V, V)+\cdots
\end{aligned}
$$

Expand $V$ in a Taylor series in $(\phi, u, I, v)$ to second order,

$$
\begin{aligned}
V= & \phi \xi_{1}+u \xi_{2}-\varepsilon I \xi_{3}+\varepsilon v \xi_{4}+\phi^{2} \boldsymbol{\eta}_{1}+\phi u \boldsymbol{\eta}_{2} \\
& +\phi I \boldsymbol{\eta}_{3}+u^{2} \boldsymbol{\eta}_{4}+\phi v \boldsymbol{\eta}_{5}+u I \boldsymbol{\eta}_{6}+u v \boldsymbol{\eta}_{7}+I^{2} \boldsymbol{\eta}_{8} \\
& +I v \boldsymbol{\eta}_{9}+v^{2} \boldsymbol{\eta}_{10}+\cdots .
\end{aligned}
$$

where $\phi, u, I, v$ are $t$ - dependent and $\boldsymbol{\eta}_{1}, \ldots, \boldsymbol{\eta}_{10}$ are $\theta-$ dependent. Substituting these expressions into (16) and equating coefficients of like quadratic powers of $(\phi, u, I, v)$ to zero results in a system of ordinary differential equations, of which we will only need five:

$$
\begin{aligned}
& \phi^{2}: 0=\mathbf{L}(\theta) \boldsymbol{\eta}_{1}+\frac{1}{2} D^{3} H\left(\boldsymbol{\xi}_{1}, \boldsymbol{\xi}_{1}\right), \\
& \phi u: 2 \mathbf{J} \boldsymbol{\eta}_{1}=\mathbf{L}(\theta) \boldsymbol{\eta}_{2}+D^{3} H\left(\boldsymbol{\xi}_{1}, \boldsymbol{\xi}_{2}\right), \\
& \phi I:-\mathbf{J} \boldsymbol{\eta}_{5}=\mathbf{L}(\theta) \boldsymbol{\eta}_{3}-\varepsilon D^{3} H\left(\boldsymbol{\xi}_{1}, \boldsymbol{\xi}_{4}\right), \\
& u^{2}: \frac{1}{2} \varepsilon \sigma \mathbf{J} \xi_{3}+\mathbf{J} \boldsymbol{\eta}_{2}=\mathbf{L}(\theta) \boldsymbol{\eta}_{4}+\frac{1}{2} D^{3} H\left(\boldsymbol{\xi}_{2}, \boldsymbol{\xi}_{2}\right), \\
& u v: \mathbf{J} \boldsymbol{\eta}_{5}+2 \varepsilon \mathbf{J} \boldsymbol{\eta}_{4}=\mathbf{L}(\theta) \boldsymbol{\eta}_{7}+\varepsilon D^{3} H\left(\xi_{2}, \xi_{3}\right) .
\end{aligned}
$$

suppressing the dependence of $D^{3} H$ on the point $\hat{U}$. Using (14), solve the 1 st, 2 nd, and 4 th equations

$$
\begin{aligned}
& \boldsymbol{\eta}_{1}=\frac{1}{2} a^{2} \hat{U}_{\theta \theta}+\alpha_{1} \xi_{1}, \\
& \boldsymbol{\eta}_{2}=a^{2} \hat{U}_{\theta \omega}+2 \alpha_{1} \xi_{2}+\alpha_{2} \xi_{1}, \\
& \boldsymbol{\eta}_{4}=\frac{1}{2} a^{2} \hat{U}_{\omega \omega}+\frac{1}{2} \varepsilon \sigma \xi_{4}+2 \alpha_{1} \xi_{3}+\alpha_{2} \xi_{2}+\alpha_{3} \xi_{1},
\end{aligned}
$$

where $\alpha_{1}, \alpha_{2}$, and $\alpha_{3}$ are arbitrary constants. Now combine the 3rd and 5th equations,

$$
\begin{aligned}
\mathbf{L}(\theta)\left(\boldsymbol{\eta}_{3}+\boldsymbol{\eta}_{7}\right)= & -\varepsilon D^{3} H\left(\boldsymbol{\xi}_{2}, \boldsymbol{\xi}_{3}\right)+\varepsilon D^{3} H\left(\boldsymbol{\xi}_{1}, \boldsymbol{\xi}_{4}\right) \\
& +2 \varepsilon \mathbf{J} \boldsymbol{\eta}_{4} .
\end{aligned}
$$

For this equation to be solvable, it is required

$$
0=\left\langle\left\langle\boldsymbol{\xi}_{1},-\varepsilon D^{3} H\left(\boldsymbol{\xi}_{2}, \boldsymbol{\xi}_{3}\right)+\varepsilon D^{3} H\left(\boldsymbol{\xi}_{1}, \boldsymbol{\xi}_{4}\right)+2 \varepsilon \mathbf{J} \eta_{4}\right\rangle\right\rangle .
$$

Substituting for $\boldsymbol{\eta}_{4}$, using (15) and noting that $\left\langle\left\langle\boldsymbol{\xi}_{1}, \mathbf{J} \boldsymbol{\xi}_{j}\right\rangle\right\rangle=$ 0 for $j=1,2,3$, 


$$
\begin{aligned}
& a^{3} \mathcal{A}^{\prime \prime}\left(\omega_{0}\right)-a^{3}\left\langle\left\langle\mathbf{J} \hat{U}_{\theta}, \hat{U}_{\omega \omega}\right\rangle\right\rangle \\
& =2\left\langle\left\langle\boldsymbol{\xi}_{1}, \frac{1}{2} a^{2} \mathbf{J} \hat{U}_{\omega \omega}+\frac{1}{2} \varepsilon \sigma \mathbf{J} \boldsymbol{\xi}_{4}\right\rangle\right\rangle .
\end{aligned}
$$

Hence with $\left\langle\left\langle\boldsymbol{\xi}_{1}, \mathbf{J} \boldsymbol{\xi}_{4}\right\rangle\right\rangle=\varepsilon$, it follows that $\sigma=$ $a^{3} \mathcal{A}^{\prime \prime}\left(\omega_{0}\right)$, confirming (6).

Properties of the homoclinic torus bifurcation.-By neglecting the higher order terms in the nonlinear normal form (5), and setting $\delta=0$, the leading order properties of the homoclinic bifurcation can be obtained

$$
\phi=\nu t-3 \frac{\nu}{\beta} \tanh (\beta t), \quad u=\nu-3 \nu \operatorname{sech}^{2}(\beta t)
$$

with $I=$ constant, $v=\varepsilon u_{t}$, and

$\nu= \pm a^{-3 / 2} \sqrt{2 I / \mathcal{A}^{\prime \prime}\left(\omega_{0}\right)}, \quad \beta=\frac{1}{2}\left(\varepsilon \nu a^{3} \mathcal{A}^{\prime \prime}\left(\omega_{0}\right)\right)^{1 / 2}$

where the sign of $\nu$ is chosen so that the latter argument is positive. The argument of the first square root in (17) is always positive since $E(\omega)=E\left(\omega_{0}\right)+\frac{\omega_{0}}{a} I+$ higher order terms. The homoclinic angle is determined from

$$
\begin{aligned}
\Delta \phi & =\int_{-\infty}^{+\infty}(u(t)-\nu) d t=-\frac{6 \nu}{\beta} \\
& = \pm \frac{(12) 2^{1 / 4}}{a^{2} \omega_{0}^{1 / 4}\left|\mathcal{A}^{\prime \prime}\left(\omega_{0}\right)\right|^{3 / 4}}\left|E(\omega)-E\left(\omega_{0}\right)\right|^{1 / 4}
\end{aligned}
$$

confirming the estimate (7). The sign of $\varepsilon E^{\prime \prime}\left(\omega_{0}\right)$ determines which $\nu$ branch in (17) is hyperbolic.

Example. - Consider the Hamiltonian system (1) on $\mathbb{R}^{4}$ with coordinates $U=\left(q_{1}, q_{2}, p_{1}, p_{2}\right)$ and

$$
H(U)=\frac{1}{2}\left(p_{1}^{2}+p_{2}^{2}\right)+V(r), \quad r=q_{1}^{2}+q_{2}^{2} .
$$

The simple form of this Hamiltonian (it is invariant under circular symmetry) is chosen because it is possible to compute all properties explicitly. It serves to illustrate the constructive aspects of the theory.

There exists a family of periodic orbits of the form

$$
\begin{gathered}
\hat{U}(\theta, \omega)=\left(\mathrm{R}(\theta) \hat{q}, \omega \mathbf{J}_{2} \mathrm{R}(\theta) \hat{q}\right), \\
\mathrm{R}(\theta):=\left[\begin{array}{cc}
\cos \theta & -\sin \theta \\
\sin \theta & \cos \theta
\end{array}\right],
\end{gathered}
$$

where $\mathbf{J}_{2}=\left.\frac{d}{d \theta} \mathrm{R}(\theta)\right|_{\theta=0}$, with $\omega$ determined by

$$
\omega^{2}=2 V^{\prime}(\hat{r}), \quad \hat{r}=\hat{q}_{1}^{2}+\hat{q}_{2}^{2} .
$$

The action is $\mathcal{A}(\omega)=\omega\|\hat{q}\|^{2}$ and so $\mathcal{A}^{\prime}(\omega)=$ $\hat{r}+\omega^{2} / V^{\prime \prime}(\hat{r})$ with $\hat{r}$ and $\omega$ related by (18). The periodic orbit is degenerate when $\hat{r} V^{\prime \prime}(\hat{r})+\omega^{2}=0$. To determine all information about the local homoclinic torus bifurcation near the degeneracy we need $\varepsilon$, the basis $\left\{\boldsymbol{\xi}_{1}, \ldots, \boldsymbol{\xi}_{4}\right\}$ and $\mathcal{A}^{\prime \prime}\left(\omega_{0}\right)$. Firstly,

$$
\hat{\xi}_{1}(\theta)=\left(\begin{array}{c}
\mathbf{J}_{2} \mathrm{R}(\theta) \hat{q} \\
-\omega \mathrm{R}(\theta) \hat{q}
\end{array}\right), \quad \hat{\xi}_{2}(\theta)=\frac{1}{2 \omega}\left(\begin{array}{c}
-\mathrm{R}(\theta) \hat{q} \\
\omega \mathbf{J}_{2} \mathrm{R}(\theta) \hat{q}
\end{array}\right)
$$

Solving the equations for $\hat{\xi}_{3}(\theta)$ and $\hat{\xi}_{4}(\theta)$,

$$
\hat{\xi}_{3}(\theta)=\frac{1}{c_{1}}\left(\begin{array}{c}
\omega \mathbf{J}_{2} \mathrm{R}(\theta) \hat{q} \\
\mathrm{R}(\theta) \hat{q}
\end{array}\right), \quad \hat{\xi}_{4}(\theta)=\left(\begin{array}{c}
c_{2} \omega \mathrm{R}(\theta) \hat{q} \\
c_{3} \mathbf{J}_{2} \mathrm{R}(\theta) \hat{q}
\end{array}\right) .
$$

with $c_{1}=-2 \omega\left(1+\omega^{2}\right), \quad c_{2}=\left(1-\omega^{2}\right) /\left(4 \omega^{3} c_{1}\right)$, and $c_{3}=\left(1+3 \omega^{2}\right) /\left(4 \omega c_{1}\right)$. We deduce that $\varepsilon=+1$ since $\left\langle\left\langle\mathbf{J} \hat{\boldsymbol{\xi}}_{4}, \hat{\xi}_{1}\right\rangle\right\rangle=\frac{1}{4} \hat{r} / \omega^{2}$ and

$$
a=\frac{2 \omega}{\sqrt{\hat{r}}}, \quad b=\frac{\left(3 \omega^{4}+2 \omega^{2}-1\right)}{4 \omega \sqrt{\hat{r}}\left(1+\omega^{2}\right)^{2}} .
$$

This provides sufficient information to construct the basis $\left\{\boldsymbol{\xi}_{1}(\theta), \ldots, \boldsymbol{\xi}_{4}(\theta)\right\}$. The remaining piece of information required is $\mathcal{A}^{\prime \prime}(\omega)$ [or equivalently $E^{\prime \prime}(\omega)$ ], and

$$
\mathcal{A}^{\prime \prime}(\omega)=\frac{\omega}{V^{\prime \prime}(\hat{r})}\left(3+\frac{\hat{r} V^{\prime \prime \prime}(\hat{r})}{V^{\prime \prime}(\hat{r})}\right) \text {. }
$$

Since $\varepsilon=+1$, the sign of $\mathcal{A}^{\prime \prime}$ determines which branch is hyperbolic. The simplest choice for $V(r)$ with the bifurcation is $V(r)=r-\frac{1}{2} r^{2}$, in which case $\omega_{0}=1 / \sqrt{3}$ and $\mathcal{A}^{\prime \prime}\left(\omega_{0}\right)=-3 \omega_{0}<0$.

*Electronic address: T.Bridges@surrey.ac.uk †Electronic address: N.Donaldson@surrey.ac.uk

[1] A. Deprit and J. Henrard, Adv. Astron. Astrophys. 6, 2 (1968).

[2] V. I. Arnold, V. V. Kozlov, and A. I. Neishtadt, Dynamical Systems III (Springer-Verlag, Berlin, 1993).

[3] J. Galan, F. J. Munoz-Almaraz, E. Freire, E. Doedel, and A. Vanderbauwhede, Phys. Rev. Lett. 88, 241101 (2002).

[4] F. J. Munoz-Almaraz, E. Freire, J. Galan, E. Doedel, and A. Vanderbauwhede, Physica (Amsterdam) 181D, 1 (2003).

[5] J. A. Sepulchre and R. S. MacKay, Nonlinearity 10, 679 (1997).

[6] K. Hirai and E. J. Heller, Phys. Rev. Lett. 79, 1249 (1997).

[7] M. Gutzwiller, Chaos in Classical and Quantum Mechanics (Springer-Verlag, New York, 1990).

[8] Y. Lan and P. Cvitanovic, Phys. Rev. E 69, 016217 (2004).

[9] T. J. Bridges, J. Fluid Mech. 505, 153 (2004).

[10] T. J. Bridges and N.M. Donaldson, report 2004 (to be published).

[11] L. Bates and J. Sniatycki, Proc. Am. Math. Soc. 114, 877 (1992).

[12] H. W. Broer, S. N. Chow, Y. I. Kim, and G. Vegter, Fields Inst. Commun. 4, 1 (1995).

[13] A. R. Champneys and G. J. Lord, Physica (Amsterdam) 102D, 101 (1997).

[14] L. Dieci and J. Rebaza, BIT 44, 41 (2004).

[15] R. H. Cushman and J. A. Sanders, Can. Math. Soc. Conf. Proc. 8, 353 (1987). 